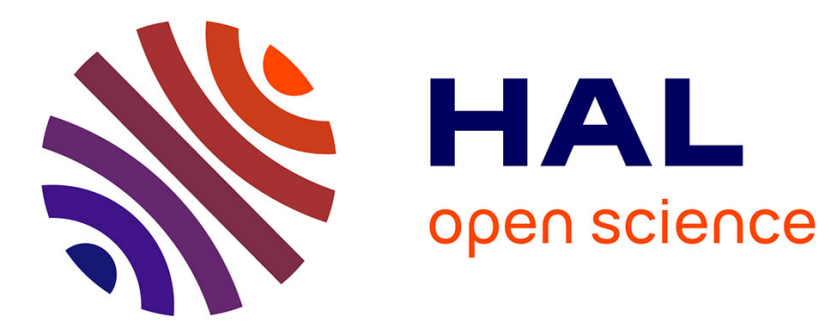

\title{
Microscopie électronique à haute tension
}

\author{
B. Jouffrey
}

\section{To cite this version:}

B. Jouffrey. Microscopie électronique à haute tension. Revue de Physique Appliquée, 1968, 3 (4), pp.307-310. 10.1051/rphysap:0196800304030700 . jpa-00242864

\section{HAL Id: jpa-00242864 https://hal.science/jpa-00242864}

Submitted on 1 Jan 1968

HAL is a multi-disciplinary open access archive for the deposit and dissemination of scientific research documents, whether they are published or not. The documents may come from teaching and research institutions in France or abroad, or from public or private research centers.
L'archive ouverte pluridisciplinaire HAL, est destinée au dépôt et à la diffusion de documents scientifiques de niveau recherche, publiés ou non, émanant des établissements d'enseignement et de recherche français ou étrangers, des laboratoires publics ou privés. 


\title{
MIGROSGOPIE ÉLEGTRONIQUE A HAUTE TENSION
}

\author{
Par B. JOUFFREY, \\ Laboratoire de Physique des Solides associé au G.N.R.S., Faculté des Sciences, 91-Orsay.
}

\begin{abstract}
Résumé. - L'auteur, après avoir rappelé brièvement le principe d'un microscope électronique par transmission, essaie de dégager quelques domaines d'application que la microscopie électronique à haute tension permettrait d'étudier efficacement. Il semble que deux questions sont à résoudre en priorité : l'étude des contrastes et celle des défauts ponctuels qui doivent être créés si la tension est assez élevée.
\end{abstract}

Abstract. - The author recalls briefly the principle of transmission electron microscopy. He tries to define some domains which could be studied by mean of the high voltage technique. He thinks that two questions have priority for study : contrasts and point defects behaviour. One question is to know if it is possible to observe point defects clusters created by high energy electrons if the energy is high enough.

I. Introduction. - Si des microscopes opérant à des tensions supérieures à $100 \mathrm{kV}$ ont été réalisés voici plus de vingt ans, c'est sous l'impulsion du Professeur Dupouy que fut construit à Toulouse voici quelques années le premier microscope à très haute tension [1 et 2]. Les performances de l'appareil, la qualité de ses images, tout en démontrant d'abord la finition du travail réalisé, ont permis de mettre en évidence tout l'intérêt d'un tel instrument pour les physiciens du solide et les métallurgistes. La grande énergie des électrons de l'ordre de $1,5 \mathrm{MeV}$ et bientôt $3,5 \mathrm{MeV}$ permet d'aborder l'étude de matériaux que l'on peut qualifier de massifs.
Avant de voir un peu plus en détail quelques domaines où l'intérêt de ce microscope semble le plus vif, je voudrais rappeler le schéma de principe d'un microscope électronique classique à transmission.

II. Principe de la microscopie électronique par transmission. - C'est pratiquement depuis la deuxième guerre mondiale que la microscopie à transmission s'est réellement développée. Au début, les tensions accélératrices utilisées dans les microscopes commerciaux étaient relativement faibles, de l'ordre de $50 \mathrm{kV}$. Ce n'est que voici une quinzaine d'années que se développèrent couramment des microscopes utilisant

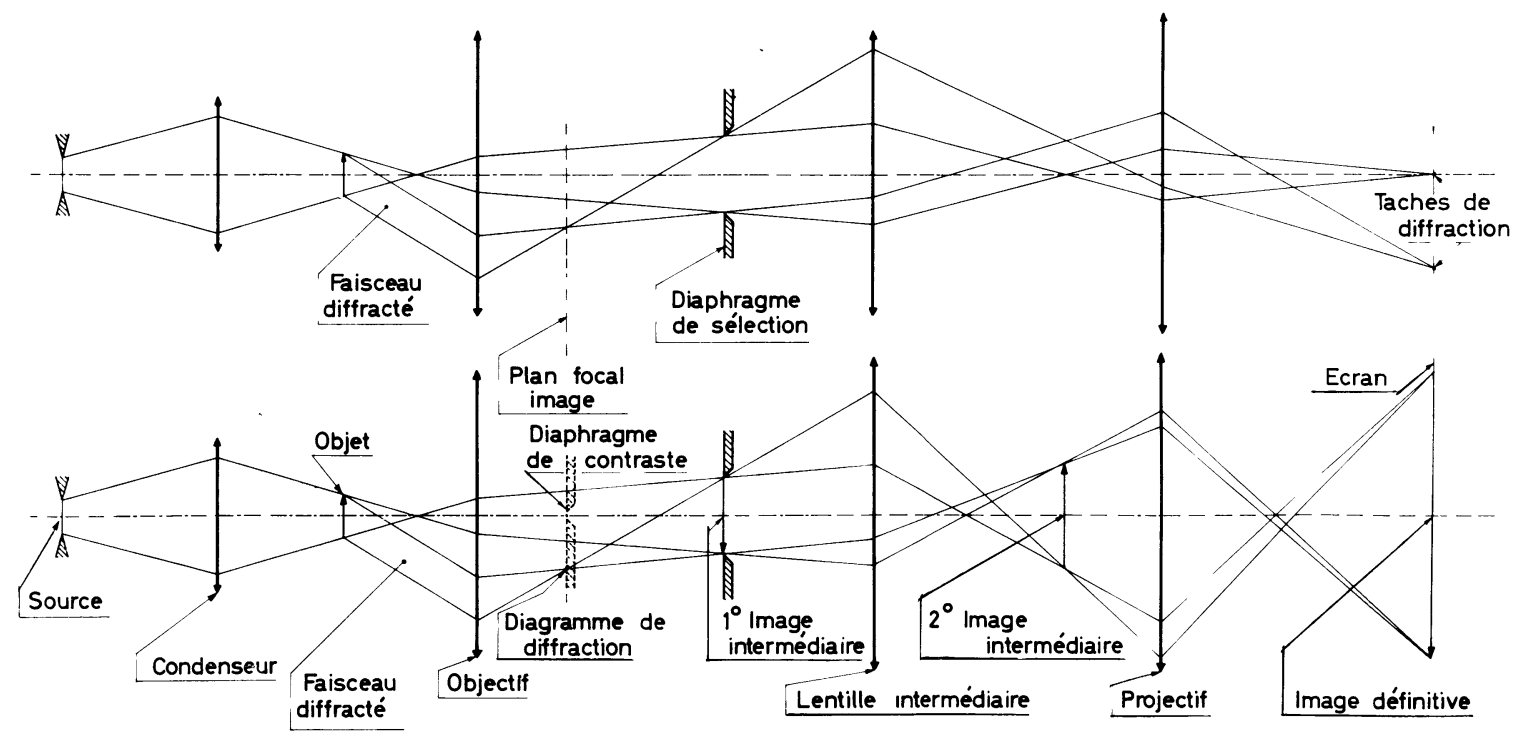

FIG. 1. 
des tensions accélératrices de $100 \mathrm{kV}$. Cet accroissement d'énergie des électrons a été déterminant dans la mise en évidence et l'étude des défauts cristallins tels que les dislocations et défauts d'empilement. Les progrès dans la réalisation de lentilles magnétiques ont amené progressivement le pouvoir de résolution sur les micrographies d'une dizaine d'angstrœems voici une dizaine d'années à 4 ou 5 angstroms pour la plupart des microscopes en vente actuellement. Le principe de l'appareil est le suivant ( $f g .1$ ).

Un filament convenablement chauffé par effet Joule et porté à une tension négative émet des électrons accélérés par une anode reliée à la masse. Un condenseur (on utilise en fait dans la plupart des cas deux condenseurs) concentre les électrons sur l'objet d'une épaisseur typique de l'ordre de $1000 \AA ̊$. Après traversée de la préparation, les électrons sont déviés par le champ magnétique d'une lentille appelée objectif qui donne de l'objet une image agrandie une vingtaine de fois. Si l'objet est cristallin, le faisceau sortant est en fait généralement divisé en plusieurs faisceaux pointant dans diverses directions et focalisés dans le plan focal image de l'objectif. C'est dans ce plan que se trouve le diagramme de diffraction permettant d'obtenir des renseignements sur les symétries du réseau cristallin de l'objet. Ces directions privilégiées suivant lesquelles on trouve des électrons obéissent à la relation de Bragg qui traduit le fait que les ondes associées aux électrons réfléchis par les plans cristallins sont en phase dans les directions déterminées par :

$$
n \lambda=2 d \sin \theta
$$

où $\lambda$ est la longueur d'onde de l'onde associée aux électrons, $d$ la distance des plans réticulaires et $\theta$ l'angle suivant lequel il y a interférence constructive, c'est-àdire suivant lequel la réflexion opère. L'angle $\theta$ vaut environ $10^{-2}$ radian en microscopie classique $\left(\sim 1 / 2^{\circ}\right)$. Le changement de l'excitation de la lentille intermédiaire placée à la suite de l'objectif permet de faire varier sa distance focale et la mise au point se fait en particulier soit sur le plan focal image de l'objectif (plan objet), donnant alors une image agrandie du diagramme de diffraction, soit sur le plan de l'image de l'échantillon donnée par l'objectif. On obtient alors l'image directe de l'objet. Une dernière lentille, le projectif, agrandit l'image de telle manière que le grossissement définitif puisse atteindre plus de 100000 . La résolution sur l'image définitive est de l'ordre de 5 à $10 \AA$. En fait, en métallurgie, les grossissements généralement utilisés sont de l'ordre de 20000 . La description que nous venons de donner correspond à la plupart des microscopes utilisés.

Le schéma de principe d'un microscope électronique à haute tension est le même que celui d'un microscope classique. Dans la pratique, seule la taille des lentilles est différente $(55 \mathrm{~cm}$ de diamètre extérieur pour l'objectif du microscope de Toulouse [2], 15 à $20 \mathrm{~cm}$ pour un microscope classique). Si un microscope électronique à $100 \mathrm{kV}$ peut être installé dans une pièce de hauteur normale, un microscope à très haute tension $\left(\sim 10^{6} \mathrm{~V}\right)$ nécessite, même avec une haute tension compacte, une hauteur libre cinq fois plus importante environ (une dizaine de mètres).

III. Microscopie à très haute tension. - Les électrons utilisés en microscopie à très haute tension sont fortement relativistes. Le tableau ci-dessous donne le rapport $\beta=\frac{v}{c}$ de la vitesse de l'électron à la vitesse de la lumière pour différentes énergies des électrons ainsi que les longueurs d'onde des ondes associées correspondantes :

\begin{tabular}{lcl}
\multicolumn{1}{c}{$500 \mathrm{kV}$} & $1 \mathrm{MV}$ & $4 \mathrm{MV}$ \\
$-\overline{-}$ & - & - \\
$0,0142 \AA$ & $0,0087 \AA$ & $0,0028 \AA$ \\
0,8629 & 0,9411 & 0,9936
\end{tabular}

la résolution sont caractérisés par le rayon du cercle de moindre confusion ( fig. 2) :

$$
R=0,61 \frac{\lambda}{\alpha}
$$

où $\lambda$ est la longueur d'onde et $\alpha$ l'ouverture du faisceau utilisé. Pour des questions d'aberration, $\alpha$ est difficilement supérieur à $10^{-3}$ radian. La résolution est, on le voit, proportionnelle à $\lambda$ et sera donc d'autant meilleure que $\lambda$ sera petit.

Ge pouvoir de résolution est théorique, mais pratiquement la résolution a une limitation provenant principalement de la construction même de la lentille

PouvoIR DE RÉSOLUTION. - Théoriquement le pouvoir de résolution est amélioré lorsque la tension croît notablement. Les effets de diffraction limitant 


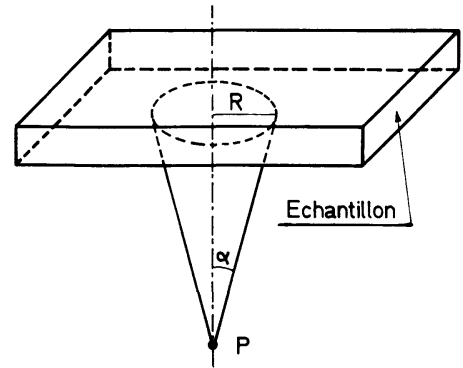

FIG. 2.

objectif. Deux types d'aberrations interviennent principalement : l'aberration sphérique et l'aberration chromatique. Le premier type d'aberration varie en première approximation comme le carré de la longueur d'onde, et l'augmentation de l'énergie des électrons jusqu'à de très hautes énergies (où la variation de $\lambda$ est lente) ne semble pas permettre d'utiliser des ouvertures beaucoup plus importantes. En revanche, il ne fait pas de doute qu'il y a intérêt d'augmenter l'énergie des électrons pour limiter les effets de l'aberration chromatique et la diminuer. La première condition est évidemment de travailler avec une haute tension très stabilisée (le microscope de Toulouse fonctionne à quelques volts près). L'aberration chromatique étant en $\frac{\Delta E}{E}$, si $\Delta E$ reste du même ordre qu'à une tension conventionnelle, il est bien évident que l'on gagne à augmenter la tension. Nous parlions quelques lignes plus haut de limitation des effets dus à l'aberration chromatique. En effet, les processus inélastiques à l'intérieur de l'échantillon donnent les pertes d'énergie observées en filtrant les électrons à la sortie de l'échantillon [3]. Ce spectre d'énergie est principalement responsable des fonds noirs à mauvaise résolution obtenus par le seul déplacement du diaphragme de contraste. L'emploi d'électrons incidents de très haute énergie diminue cet effet car le libre parcours moyen des électrons est plus grand. Les angles de Bragg plus petits qu'en microscopie traditionnelle tendent également à améliorer les fonds noirs (faisceaux moins écartés de l'axe), mais cet effet est petit en comparaison de l'effet chromatique. On peut donc dire, ce qui est très important, que la possibilité d'observer des échantillons avec un tel microscope ne se fait pas au détriment de la qualité des images.

III.2. ÉPAISSEUR UTILISABLE DES ÉGHANTILLONS. Les auteurs donnent en général une loi parabolique (voir fig. 3) :

$$
t \propto \beta^{2} .
$$

Les résultats que nous donnons sont ceux que Uyeda et Nonoyama [4] ont obtenus pour de la molybdénite. On voit que si l'épaisseur utilisable à $700 \mathrm{kV}$ est $1,5 \mu$ à $1200 \mathrm{kV}$ cette épaisseur atteint $6 \mu$. Ces auteurs trouvent cependant que vers $1200 \mathrm{kV}$ la valeur obtenue est légèrement supérieure à celle donnée par la loi parabolique (fig. 3).

Auparavant, d'autres auteurs $[5,6,7]$ avaient mené

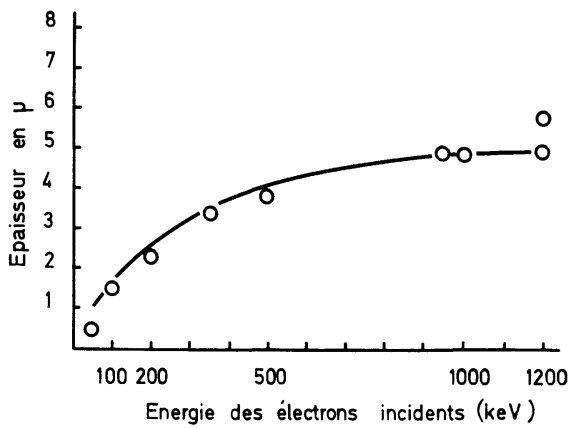

FIG. 3.

des études sur ce point très important, certains menant leurs études avec des électrons d'énergie aussi élevée que $1200 \mathrm{keV}$.

III.3. Préparation des objets. - Cette préparation se fait selon les méthodes classiques, c'est-à-dire par l'une des méthodes suivantes : polissage électrolytique ou dissolution chimique, bombardement ionique, décharge électrique, évaporation, etc.

III.4. Domaines possibles D'utilisation. - L'utilisation d'électrons d'une énergie de $1200 \mathrm{keV}$ au lieu d'une énergie de $100 \mathrm{keV}$ permet, nous l'avons vu, de multiplier par un facteur important l'épaisseur utilisable des échantillons. On peut donc espérer étudier des matériaux pratiquement massifs et d'autant plus qu'ils sont plus légers. Un grand nombre d'applications peuvent être alors envisagées :

1) Comparaison des résultats obtenus avec ceux fournis par la méthode de Lang aux rayons $\mathrm{X}$ dans des cristaux contenant peu de dislocations, la microscopie présentant l'avantage de permettre une étude continue d'un échantillon d'une épaisseur nulle à quelques microns.

2) Étude des structures de dislocations de taille inférieure à l'épaisseur des lames cristallines (tous les réseaux d'écrouissage). Cette étude à trois dimensions nécessite encore plus que dans la microscopie classique la technique de la stéréoscopie. On pourra avec intérêt comparer les structures entre les parties minces et épaisses des échantillons (formation des cellules). L'étude de la polygonisation et de la recristallisation pourra également être développée avec intérêt.

3) Déformations in situ permettant d'étudier :

- le comportement des dislocations lorsque après traction on relâche les contraintes (étude de la stabilité de l'état écroui),

- vitesse des dislocations,

- nucléation des macles, problèmes liés aux transformations martensitiques, 
- interactions entre dislocations et petits précipités de taille inférieure à celle de la lame,

- énergies des défauts d'empilement.

4) Étude des lames en épitaxie, ces lames étant assez épaisses pour que l'épitaxie soit facilement réalisée.

5) Mesure des coefficients de diffusion en surface par étude de l'évolution de rayures de surface durant un recuit.

6) Cinétiques de croissance de précipités ou de bulles de gaz ou de disparition de boucles ou dipôles de dislocations en fonction de l'épaisseur de l'échantillon.

7) Étude de lames minces de gaz rares condensés sur support amorphe ou cristallin.

8) Problèmes d'implantation d'atomes étrangers dans l'échantillon et en particulier étude de la « canalisation » des ions dans un cristal, la pénétration pouvant alors atteindre dans de bonnes conditions quelques microns pour des énergies des ions primaires de l'ordre de $10 \mathrm{keV}$. La microscopie se prête particulièrement bien à ces études, car il est possible de localiser les amas de défauts ponctuels dans l'épaisseur de l'échantillon à mieux que $100 \AA$ près.

9) Étude directe des lames obtenues par trempe ultra-rapide à partir du liquide. Le polissage de ces échantillons est compliqué et la microscopie classique ne permet de voir que leur bord.

10) Étude des domaines et parois magnétiques en fonction de l'épaisseur (comparaison avec la méthode de Lang), mais également en fonction de la température.

11) Étude de la ferro-électricité.

12) Étant donné la plus grande vitesse des électrons, les diffusions inélastiques seront moins importantes qu'en microscopie classique. Le libre parcours moyen des électrons est plus grand et il semble que la décomposition de substances organiques sous le faisceau se produira après des temps d'observation plus longs. Cependant, avant d'être à même d'affirmer qu'il est possible d'utiliser la microscopie à haute tension pour l'étude de cinétiques par exemple, il faudrait être sûr que la formation de défauts ponctuels n'est pas importante [8].

On peut facilement imaginer d'autres applications de cette technique, mais nous avons vu tout l'intérêt qu'elle présente pour les physiciens du métal et les métallurgistes.

Tous ces projets d'études nécessiteront des mises au point d'accessoires spécialement conçus pour le microscope utilisé, mais dont les principes ne diffèrent pas de ceux des accessoires utilisés en microscopie classique.

III.5. INTERPRÉTATION DES CONTRASTES. - Il reste que l'interprétation des micrographies électroniques présente souvent des difficultés plus grandes que celles rencontrées à plus basse tension pour lesquelles l'étude des images nécessite déjà des théories très élaborées. En effet, si normalement le microscopiste n'a pas trop de difficultés, pour un échantillon d'épaisseur convenable, à obtenir des diagrammes de diffraction à deux ondes (deux spots de diffraction), le plus grand rayon de la sphère d'Ewald $\left(\right.$ rayon $\left.\frac{1}{\lambda}\right)$ lié à un plus petit angle de Bragg $\left(\sim \frac{10}{10}\right)$ entraîne dans de nombreux cas la présence de $N$ ondes ( $N$ spots de diffraction) et les interprétations en sont alors encore plus compliquées. Il y a donc intérêt à développer des études théoriques sur les contrastes obtenus lorsque $N$ ondes opèrent. Notons que des travaux dans ce domaine ont déjà été réalisés en particulier en Angleterre.

IV. Conclusion. - Depuis la réalisation du microscope de Toulouse, les constructions récentes en Angleterre à Cambridge, au Japon par les firmes Hitachi, Jeol et Shimadzu, aux États-Unis par R.C.A. montrent s'il en est encore besoin tout l'intérêt que le développement de cette technique soulève.

L'utilisation de la haute tension aura certainement un champ de possibilités nouvelles très étendues pour le microscopiste proprement dit, le physicien du solide et le métallurgiste. L'intérêt de l'étude des interactions des électrons de haute énergie avec l'échantillon est évident pour le physicien du solide. L'étude de création des défauts ponctuels, si la tension est assez élevée, spécialement dans le cas des métaux légers, ou de perturbations dans les polymères ou objets biologiques, est sans doute le premier domaine à compléter. Ge domaine de recherche est d'une grande importance pour les métallurgistes, en particulier pourl'étu de des précipitations et des mouvements de dislocations.

\section{BIBLIOGRAPHIE}

[1] Dupouy (G.), Perrier (F.) et Fabre (R.), $C . R$. Acad. Sc., 1961, 252, 627.

[2] Dupouy (G.) et PerRier (F.), J. Microscopie, 1962, 1, 167-192.

[3] Castaing (R.) et Henry (L.), C. R. Acad. Sc., 1962, 255, 76-78.

[4] Uyeda (R.) et Nonoyama (M.), Japanese J. Appl. Phys., 1968, 7, 3, 200.
[5] Hashimoto, J. Appl. Phys., 1964, 35, 277.

[6] Dupouv (G.), Perrier (F.), Uveda (R.), Ayroles (R.) et MAZEL (A.), J. Microscopie, 1965, 4, 429.

[7] Fujita (H.), Kawasaki, Furubayashi (E.), KajiWara (S.) et TaOka (T.), J. Appl. Phys., 1967, 6, 214.

[8] Makin (M. J.) et Sharp (J. V.), J. Mat. Sc., 1968, 3, 360 . 